\title{
The Hanger Reflex: An Inexpensive and Non-invasive Therapeutic Modality for Dystonia and Neurological Disorders
}

\author{
Takashi ASAHI ${ }^{1}$, Takuto NAKAMURA ${ }^{1}$, Michi SATO², Yuki KON², \\ Hiroyuki KAJIMOTO ${ }^{2}$, and Shuji $\mathrm{SATO}^{2}$ \\ ${ }^{1}$ Department of Neurosurgery, Kanazawa Neurosurgical Hospital, Nonoichi, Ishikawa, Japan \\ ${ }^{2}$ Department of Informatics, The University of Electro-Communications, Chofu, Tokyo, Japan
}

\begin{abstract}
The hanger reflex is a phenomenon characterized by the involuntary rotation of the head when a wire hanger is worn around the head such that a force is applied to the frontal temporal area by the longer side of the hanger. The application of a shearing force on the skin is thought to be the cause of this phenomenon. Attempts have been made to treat cervical dystonia using equipment designed to induce the hanger reflex. This reflex may have implications in the treatment of headaches, cervical pain, and adhesive capsulitis. The hanger reflex is seen not only in the head region but is also in other parts of the body. Thus, it could be used in the treatment of systemic dystonias. The hanger reflex may help develop inexpensive and non-invasive treatment for dystonia or other neurological diseases and is expected to be the focus of research in the future.
\end{abstract}

Keywords: dystonia, torticollis, chronic headache, shoulder stiffness

\section{Introduction}

The hanger reflex is a phenomenon, which is characterized by the involuntary rotation of the head when a wire hanger is worn around the head such that a force is applied to the frontal temporal area by the longer side of the hanger (Fig. 1, Movie 1. Movies 1-5 are available online.). ${ }^{1-3)}$ This phenomenon first came to light publicly in 1995, when a television program in Japan reported that a man aimlessly wore a wire hanger around his head while he was studying and found that his head rotated. After publication in a book, this phenomenon was termed as the "hanger reflex" by Kajimoto et al. at the University of Electro-Communications in 2008. This reflex subsequently became a topic of research. ${ }^{1)}$

A medical study conducted outside Japan and published in 1991 reported that wearing a square cardboard box on the head caused the head to rotate, resulting in symptomatic improvement in patients

Received May 12, 2020; Accepted July 20, 2020

Copyright $\subseteq 2020$ by The Japan Neurosurgical Society This work is licensed under a Creative Commons AttributionNonCommercial-NoDerivatives International License. with spasmodic torticollis. ${ }^{4)}$ This report appears to describe a similar phenomenon. However, no related study has been published on this topic since then. In this study, we reviewed the research conducted so far and discussed the future outlook of the application of the hanger reflex.

This study was approved by the Ethics Committee of the University of Toyama (ID 23-141) and Kanazawa Neurosurgical Hospital (ID 27-03, 27-04). Written informed consent was obtained from all subjects prior to participation in the study.

\section{Application in Cervical Dystonia}

Research on the hanger reflex began in 2009, when the first author chanced upon a television program reporting that this phenomenon was being researched at the University of Electro-Communications. The scene was spectacular-an entertainer worn a wire hanger around the head, which started rotating. Based on the TV program, a wire hanger was placed around the head of a patient with cervical dystonia, whose symptoms had persisted even after surgical treatment. Astoundingly, the abnormal head rotation ceased immediately after the application. ${ }^{3)}$ Nevertheless, the continuous use of wire hangers is 
A

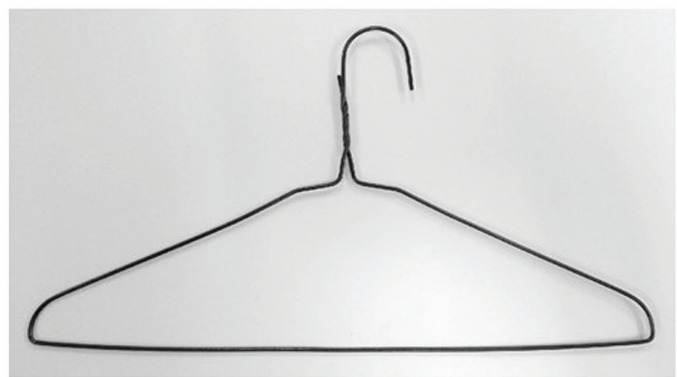

B

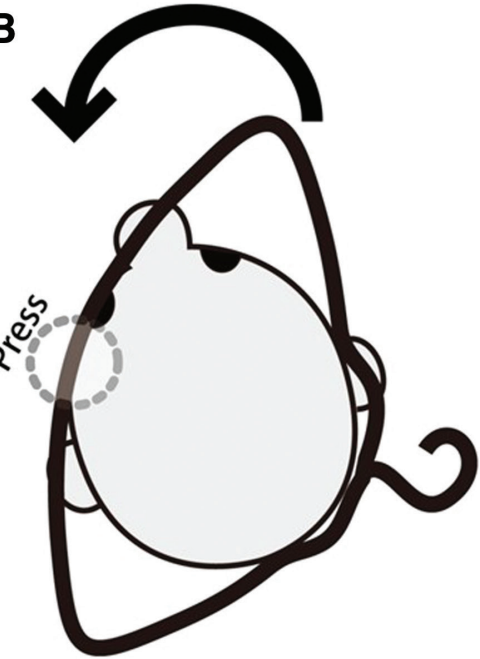

Fig. 1 Hanger reflex. (A) A wire hanger used to induce the hanger reflex and (B) the hanger is stretched out and worn around the head, touching the frontotemporal area and causing a sensation of the head rotating toward the compressed side. These figures have been reproduced from previously published papers. ${ }^{2,6)}$

A

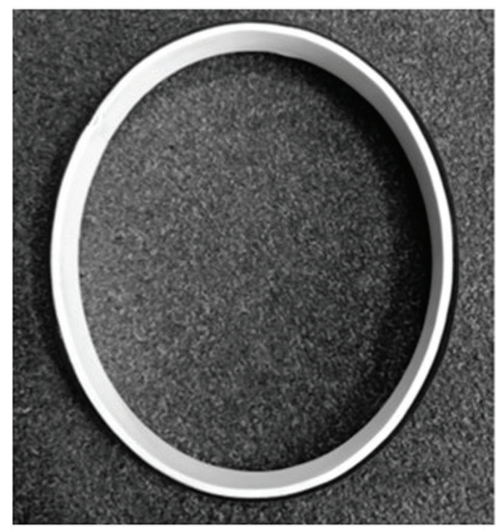

B
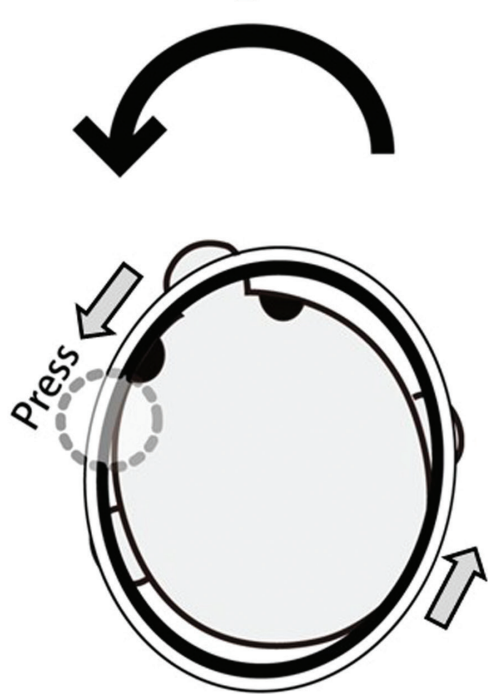

C

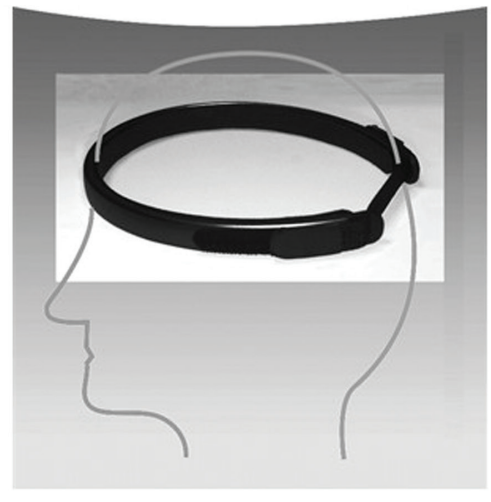

Fig. 2 A device to induce the hanger reflex for rotation. (A) Equipment that triggers the hanger reflex and (B) the hanger reflex occurs toward the compressed side when the device is rotated while compressing the frontotemporal area. These figures have been reproduced from previously published papers. ${ }^{2,6)}$ (C) Rakubi (rotational type) (TSS, Tokyo, Japan) General medical device in Japan-non-active simplified traction device (35519001).

impractical, which raised the need for an esthetically acceptable portable device. Research for developing such a device was launched in collaboration with the University of Electro-Communications (Figs. 2A and 2B). ${ }^{5}$ Head rotation became controllable even in the absence of a wire hanger in patients who used this device after 1 month. ${ }^{3)}$ A study was to demonstrate the effect of the hanger device commenced in 2010, and a multicenter clinical research trial began in 2012.6) The device was used for 30 minutes/day for 3 months in these studies and the symptoms were assessed before the start of device use and 3 months after device use. Data on rotational cervical dystonia were compiled from 19 patients, which indicated that a significant reduction (improvement) was observed in the Toronto Western Spasmodic Torticollis Rating Scale (TWSTRS) part I scores at 3 months. ${ }^{6}$ ) 

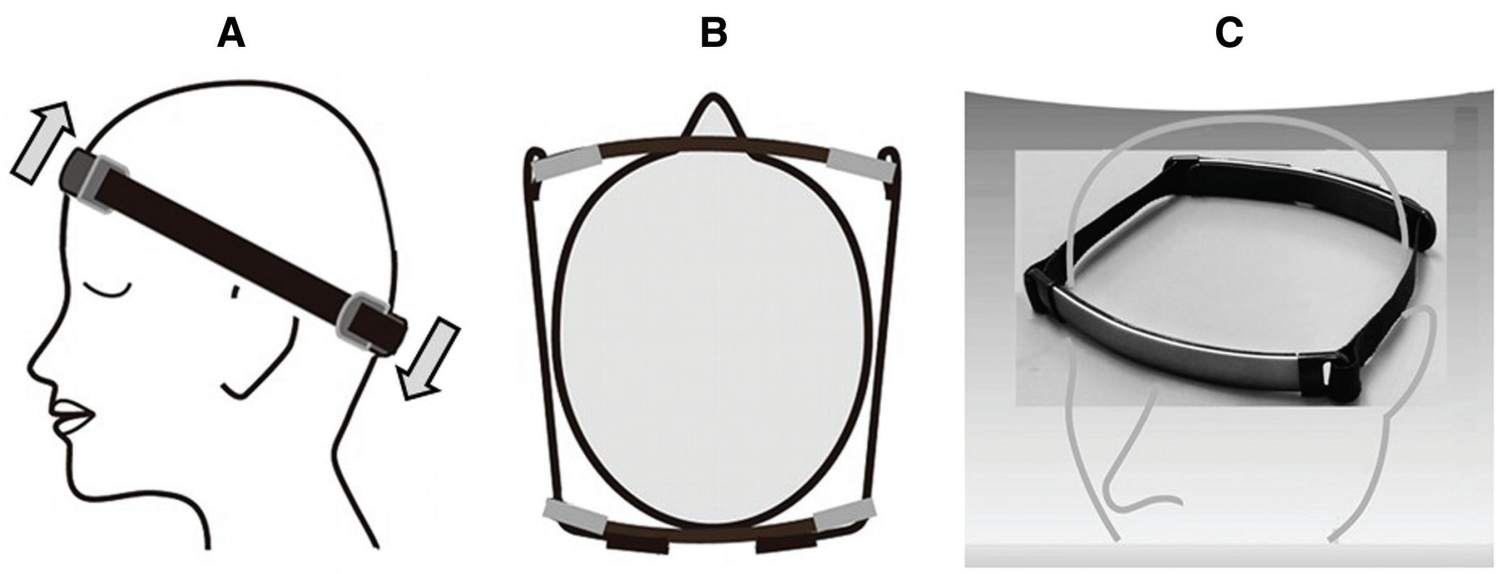

Fig. 3 A device to induce the hanger reflex for backward-forward rotation. (A) Equipment that triggers the hanger reflex and (B) the hanger reflex occurs toward the upper side when the device is rotated while compressing the upper frontal area. (C) Rakubi 2 (antero-posterior type, TSS, Tokyo, Japan).

Continual use after 3 months proffered further improvement in symptoms. The device that induces the hanger reflex has since been improved and is currently distributed commercially as a medical device in Japan and Korea (Fig. 2C). Herein, we present the case of a patient with cervical dystonia treated with this device (Movie 2). The patient was 64-year-old woman. Her head turned to the right for 5 years. She tried several medications, which proved ineffective. She could turn her head to the left easily on wearing the device on her head. She wore the device once a day for 30 minutes. Three months later, her abnormal head movement resolved almost completely even without wearing the device. The TWSTRS part I score decreased from 13 to 1 after 3 months. There was no recurrence 9 months after the trial. It is understood that the hanger reflex occurs not only for rotational movement but also for backward-and-forward movements, and a device for the latter type of movement is also commercially available (Fig. 3C). This device was also found to be effective for patients with a forward head movement pattern (i.e., dropped head syndrome). We present a case of anterocollis. The patient was a 55-year-old woman, with a dropped head and had trouble performing activities of daily living. She was referred to our hospital because her symptoms did not improve with medication. The range of movement expanded when the wire hanger was placed in the backward-forward direction. The movement in the backward-bending and rotational directions showed immense improvement. The symptoms completely improved after 6 months when the hanger device was worn backward-forward, and were alleviated completely without any other interventions (Movie 3).

\section{Application in Treating Headache, Neck Pain, and Shoulder Stiffness}

Headaches induced by cervical dystonia are described in the International Classification of Headache Disorders. Cervical dystonia may be undiagnosed in patients with chronic headaches and frozen shoulder. ${ }^{7,8}$ Botulinum toxin is in fact effective for treating various headaches. ${ }^{9,10)}$ We present the case of a 20-year-old woman with chronic headaches since junior high school (Movie 4). She had a past history of right shoulder dislocation. She visited several doctors for the treatment of chronic shoulder pain and headaches, but her symptoms exacerbated gradually. Finally, she came to our clinic. Her condition was characterized by migraines and medication overuse headaches. She also experienced severe restriction in the range of head movement. The hanger device was used to treat her anterocollis, which resulted in a dramatic increase in the range of head, immediately after application. She used the device every day for 3 months. Each trial lasted up to 30 minutes and she was allowed to wear the device as often as she wished. The range of head movement improved even without wearing the device after 3 months. The combination of hanger therapy with conventional therapy for headaches resulted in an improvement in the Headache Impact Test-6 (HIT-6) score from 70 (before treatment) to 44 (after 6 months).

\section{Application to Body Parts Besides the Head}

Researchers found that the hanger reflex occurs not only around the head region but also around the wrists, ankles, and lumbar area. ${ }^{11-13)}$ We developed a device that induced the hanger reflex on the wrist 
A

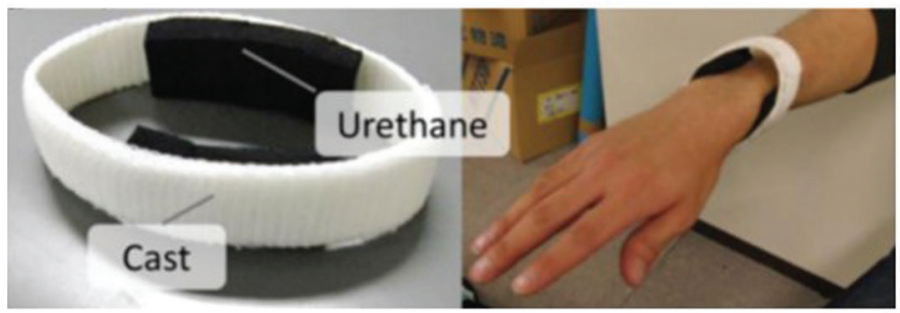

B

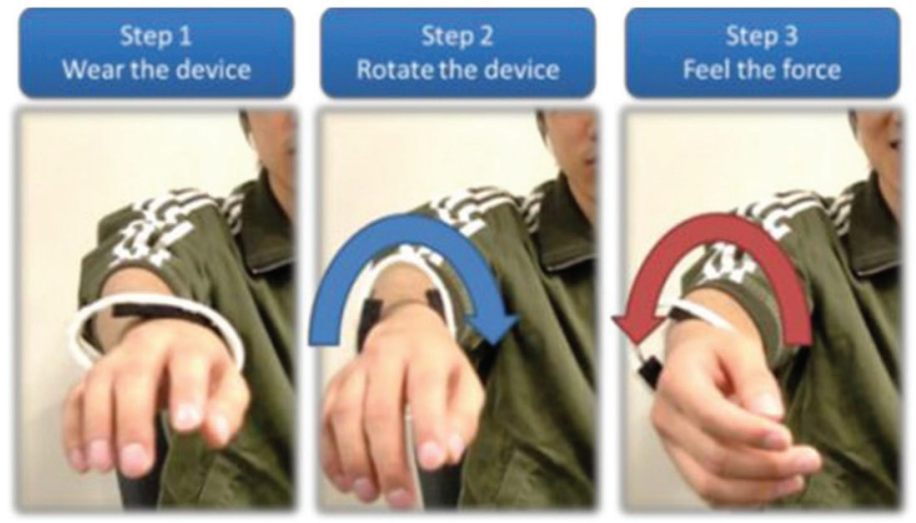

C

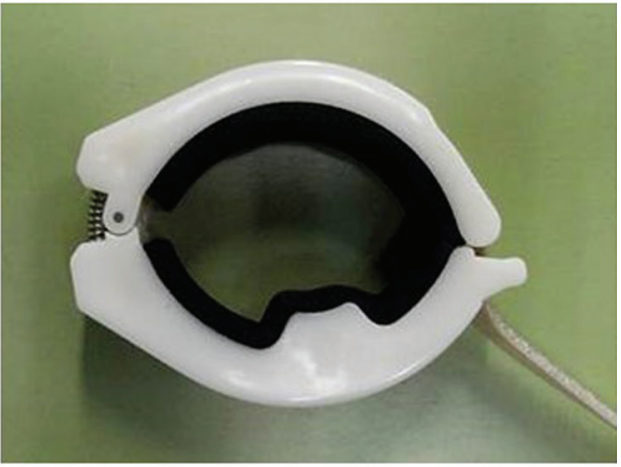

D

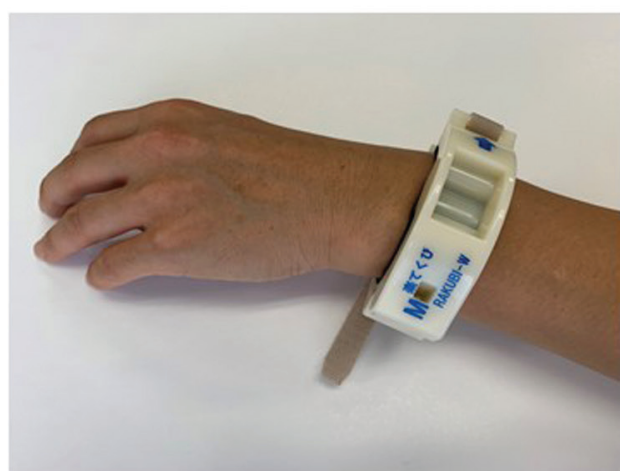

Fig. 4 A device induces the hanger reflex on the wrist: (A) This device induces the hanger reflex, (B) this figure shows the method to induce the hanger reflex on the wrist. The figure have been reproduced from previously published papers. ${ }^{13)}$ (B and C) Ratekubi (TSS, Tokyo, Japan): a device that induced the hanger reflex on the wrist.

(Fig. 4). We used the device in a patient with upper limb local dystonia (Movie 5). A 30-year-old man with bilateral focal hand dystonia (writer's cramp) underwent left thalamotomy for the right upper limb symptom. The symptoms in his right hand disappeared while those in his left hand remained. His forearm pronated while using chopsticks. The pronation improved within 1 week of using the device to induce the hanger reflex to induce supination of the forearm. Pronation while eating with chopsticks improved completely even without using the device. The symptoms did not recur for 4 months since then.

\section{Mechanism of Action of the Hanger Reflex}

An examination of the incidence of the hanger reflex in 120 healthy participants revealed that it was present in approximately $90 \%$ of the participants. ${ }^{2)}$ Sensory tricks, such as touching the face, are well-known phenomena that can temporarily relieve the symptoms of dystonia. We do not believe that the hanger reflex is a sensory trick because the hanger reflex is present in normal individuals and patients with dystonia. The application of shearing force to the skin is thought to be the mechanism underlying this reflex. This hypothesis is supported by the work of our coresearchers, who developed a spring-loaded lozenge device to generate a shearing force to the skin of the head. ${ }^{14)}$ They found that the head rotated medially when the skin was sheared to the medial side and laterally when the skin was sheared to the lateral side, irrespective of the region of frontotemporal compression. Furthermore, they found that both the peak value and integral value of skin deformation were highly correlated with the subjective strength of the hanger effect. ${ }^{14)}$ Thus, these results suggest that the hanger reflex is triggered toward the direction in which the skin is sheared. The skin of the frontal temporal area deviates outward from the temporal line but is impeded by friction when a wire hanger is worn on the head. In this condition, the individual may feel an urge to turn their head in the direction of the deviation to resolve the discomfort, but the skin keeps deviating even when the head is turned. Eventually, the head rotates to the side of the deviation. Head rotation with our device first pulled the skin over the frontotemporal region to the medial side and subsequently pulled the skin laterally after the device was released (Fig. 2). We believe that the hanger 
reflex is a type of sensory illusion induced by skin sensation, where the individual believes that continuing to turn their head will relieve the discomfort. ${ }^{14)}$

It is known that the hanger reflex can occur not only in a rotational direction but also in the frontal, backward, and lateral directions, due to similar underlying mechanisms. Furthermore, this phenomenon can be induced in other parts of the body besides the head by the same mechanism. ${ }^{11-13)}$

\section{Future Prospects}

Some patients were completely cured with the devices that induced the HR, as shown in this study. Dystonia is considered to be a network disorder. ${ }^{15)}$ It is thought that the abnormal network was modified and reconstructed through the skin sensation induced by the devices in patients who experienced complete recovery.

However, the devices were not effective for all cases. First, the HR did not appear in some cases. In the previous study, the HR did not appear in approximately $10 \%$ of healthy participants. ${ }^{16)}$ The shape of the head of patients who did not respond to these devices was less likely to generate shear force to the skin. Furthermore, some patients also underwent additional treatment with pharmacotherapy, botulinum toxin injection, and deep brain stimulation because their symptoms did not improve sufficiently with the device. A study has shown that the HR is enhanced by adding vibrations to the device. ${ }^{12)}$ Devices under development are expected to have this feature in the future.

The hanger device could be in high demand in the future, especially in developing countries, where highly expensive therapeutic treatments, such as botulinum toxin and deep brain stimulation are not easily accessible. Thus, there is a plan to expand the availability of this device to foreign countries. The hanger reflex is an intriguing phenomenon that may offer treatment solutions not only for conditions related to the head as shown in this report but may also be effective in treating dystonia of other parts of the body besides the head and neck. The hanger reflex may inspire inexpensive and non-invasive treatments for dystonia and is expected to be the focus of research in the future.

\section{Acknowledgment}

The authors wish to thank to Dr. Genko Oyama of Juntendo University, Dr. Akito Hayashi Juntendo of University Urayasu Hospital, Dr. Kazunori Tanaka of Saiseikai Matsuyama Hospital, Dr. Shunya Nakane of Kumamoto University, Dr. Junichi Miyahara and Dr. Takao Takeshima of Tominaga Hospital, Dr. Masami
Fujii of Yamaguchi Prefectural Grand Medical Center, Dr. Masaaki Higuchi of Fukuoka University, and Dr. Shiro Horisawa and Dr. Takaomi Taira of Tokyo Women's Medical University for participating in the clinical trials and discussing and advising us about the hanger reflex.

\section{Conflicts of Interest Disclosure}

There are no potential conflicts of interest associated with this research which did not receive direct financial funding from any source.

\section{References}

1) Matsue R, Sato M, Hashimoto Y, Kajimoto H: "Hanger reflex": a reflex motion of a head by temporal pressure for wearable interface. SICE Annual Conference: 1463-1467, 2008

2) Asahi T, Sato M, Kajimoto H, Koh M, Kashiwazaki D, Kuroda S: Rate of hanger reflex occurrence: unexpected head rotation on fronto-temporal head compression. Neurol Med Chir (Tokyo) 55: 587-591, 2015

3) Asahi T, Hayashi N, Hamada H, et al.: Application of the hanger reflex to the treatment of cervical dystonia. Funct Neurosurg 49: 173-176, 2010 (Japanese)

4) Christensen JE: New treatment of spasmodic torticollis? Lancet 338: 573, 1991

5) Kajimoto $H$, Sato $M$, Iguchi $T$, Asahi T: Orthosis. World. WO 2016/052400 A1: 2016 Apr 7

6) Asahi T, Sato M, Nakamura T, et al.: Pilot study of a device to induce the hanger reflex in patients with cervical dystonia. Neurol Med Chir (Tokyo) 58: 206-211, 2018

7) Ondo WG, Gollomp S, Galvez-Jimenez N: A pilot study of botulinum toxin A for headache in cervical dystonia. Headache 45: 1073-1077, 2005

8) Bezerra ME, Rocha-Filho PA: Headache attributed to craniocervical dystonia - a little known headache. Headache 57: 336-343, 2017

9) Gady J, Ferneini EM: Botulinum toxin A and headache treatment. Conn Med 77: 165-166, 2013

10) Finkel AG: Botulinum toxin and the treatment of headache: a clinical review. Headache 51: 15651572, 2011

11) Kon Y, Nakamura T, Kajimoto H: Interpretation of navigation information modulates the effect of the waist-type hanger reflex on walking IEEE Symposium on 3D User Interfaces: 107-115, 2017

12) Nakamura T, Kajimoto H: Enhancement of perceived force from the hanger reflex on head and ankle by adding vibration. in Hasegawa $\mathrm{H}$ et al. (eds): Haptic Interaction Asia Haptics 2016 Lecture Notes in Electrical Engineering, vol 432 Singapore, Springer, 2016, pp 278-280

13) Nakamura T, Nishimura $N$, Sato $M$, Kajimoto $H$ : Application of hanger reflex to wrist and waist. IEEE Virtual Reality (VR): 181-182, 2014 
14) Miyakami M, Kon Y, Nakamura T, Kajimoto H: Optimization of the hanger reflex (I): examining the correlation between skin deformation and illusion intensity. In: Prattichizzo D, Shinoda H, Tan H, Ruffaldi E, Frisoli A (eds). Haptics: Science, Technology, and Applications EuroHaptics 2018 Lecture Notes in Computer Science, vol 10893, Cham, Springer, 2018, pp 36-48

15) Schirinzi T, Sciamanna G, Mercuri NB, Pisani A: Dystonia as a network disorder: a concept in evolution. Curr Opin Neurol 31: 498-503, 2018
16) Asahi T, Hayashi N, Sato M, et al.: The occurrence rate of the hanger reflex in healthy volunteers. In: 15th international congress of Parkinson's disease and movement disorders. Toronto, 2011.

Corresponding author: Takashi Asahi, MD, PhD Department of Neurosurgery, Kanazawa Neurosurgical Hospital, 262-2 Gomachi, Nonoichi, Ishikawa 921-8841, Japan.

e-mail: takashi-tym@umin.ac.jp 